\title{
Immunogenicity of hepatitis B vaccine in preterm infants
}

Orna Blondheim, David Bader, Martha Abend, Marina Peniakov, Danny Reich, I Potesman, Rachel Handsher, Ifat Gidoni, Nehama Linder

\begin{abstract}
Aim-To assess the immunogenicity of hepatitis $B$ vaccine in preterm and term infants, given in a sequence of three doses beginning soon after birth.

Method-The immunogenicity of hepatitis $B$ vaccine was assessed in 176 preterm infants ( $<35$ weeks of gestation), immunised soon after birth, and compared with that in 46 term infants. Titres of hepatitis $B$ antibodies were determined one to two months after the third vaccine. The significance of the differences between the term and preterm groups was determined using Student's $t$ test.

Results-A similar proportion of infants in both preterm and term groups attained protective titres of hepatitis $B$ antibodies (88.7\% vs $93.4 \%$, respectively; $p=N S)$. However, the term infants had a higher geometric mean titre of antibodies after the third vaccine than did the preterm infants $\mathbf{( 7 0 1 . 2}$ (745.0) vs $469.1(486.2) \mathrm{mU} / \mathrm{ml}$, respectively; p<0.03).

Conclusion-Hepatitis $B$ vaccine is effective in most preterm infants when given soon after birth. It may be advisable to determine the immune response at 12-24 months of age to booster the non-responders.
\end{abstract}

(Arch Dis Child Fetal Neonatal Ed 1998;79:F206-F208)

Keywords: hepatitis B vaccine; immune response

Departments of Neonatology, Ha'

Emek, Bnei Zion and Chaim Sheba (Tel Hashomer) Medical Centers,

Technion Institute of

Technology, The B.

Rappaport School of

Medicine,

Haifa,

Sackler School of

Medicine, Tel Aviv

University, Israel

O Blondheim

D Bader

M Abend

M Peniakov

D Reich

I Potesman

R Handsher

I Gidoni

$\mathrm{N}$ Linder

Correspondence to: Dr Orna Blondheim Department of Neonatology, Haemek Medical Center,

Afula,

Email:

blond@netvision.net.il

Accepted 3 April 1998

The efficacy and safety of recombinant DNA hepatitis B vaccine in adults, children, and term neonates is well known. However, few data are available on its efficacy in preterm infants..$^{1-5}$ The current recommendation is to immunise preterm infants at the appropriate age without correction for prematurity. ${ }^{6}$ It is unclear whether the immature immune system of preterm infants can respond adequately to hepatitis $\mathrm{B}$ vaccine when the first dose is given soon after birth.

This multicentre study aimed to assess the immunogenicity of hepatitis $B$ vaccine in preterm infants soon after birth and to compare it with that of term infants.

\section{Methods}

Two hundred preterm infants (23-35 weeks of gestational age), born to mothers negative for hepatitis B surface antigen (HBsAg), were eligible for the study. Fifty term infants $(>37$ weeks gestational age) were enrolled as controls. Infants were enrolled at the three participating medical centres between January 1992 and January 1993 (stage 1) and April 1993 and July 1993 (stage 2) after parental consent had been obtained. The study was approved by the ethics committees of the participating centres and of the Health Ministry.

Infants were vaccinated three times with the recombinant hepatitis B vaccine Engerix B (SmithKline Beecham Biologicals, Rixensaart, Belgium) at the recommended dose of $10 \mathrm{mcg} /$ $0.5 \mathrm{ml}$. The first dose was given as soon as possible after birth; a serious clinical condition caused a delay in some cases. The second and third doses were given one and five months later, respectively. Exclusion criteria included delayed first dose of hepatitis $B$ vaccine $>2$ weeks of age, major congenital anomalies, or treatment with immunoglobulin.

Blood samples were drawn 1-2 months after the third vaccine. The blood was allowed to clot and the serum was separated and stored at a temperature of $-70^{\circ} \mathrm{C}$ until analysed. Antihepatitis B antibody titres and HBsAg were determined using commercial radio immunoassay kits (Abbot Laboratories, Chicago, Illinois). In stage 1 the antibody titre was determined only up to $150 \mathrm{mU} / \mathrm{ml}$ and reported as $>150$ when higher. In stage 2 dilutions were also above $150 \mathrm{mU} / \mathrm{ml}$. Immunity was defined as an hepatitis B antibody titre of $10 \mathrm{mU} / \mathrm{ml}$.

Student's $t$ test was used to compare the means of each parameter between groups. The $\chi^{2}$ test was used to compare the distribution of categorical variables between groups. A p value of 0.05 or less was regarded as significant.

\section{Results}

One hundred and seventy six preterm infants $(88 \%)$ completed the study, 105 in stage 1 and 71 in stage 2 . Their clinical data are presented in table 1 . Twenty four infants did not complete the study due to: death, non-compliance, and loss to follow up. They were the same as the ones that did complete the study in terms of birthweight, gestational age, Apgar scores or incidence of respiratory distress syndrome. Forty six term infants (92\%) completed the study and their data are presented in table 2 .

Table 1 Characteristics of preterm infants

\begin{tabular}{llll}
\hline & Responders & Non-responders & p Value \\
\hline Number of patients & 156 & 20 & \\
Male/female & $76 / 80$ & $12 / 8$ & NS \\
Gestational age (weeks) & & & \\
$\quad$ Mean & $31.9(2.1)$ & $31.4(2.5)$ & NS \\
$\quad$ Range & $24-35$ & $23-34$ & NS \\
Birthweight (kg) & $1.56(0.4)$ & $1.53(0.5)$ & NS \\
\hline
\end{tabular}


Table 2 Characteristics of term infants

\begin{tabular}{lll}
\hline & Enrolled & Completed \\
\hline Number of patients & 50 & 46 \\
Male/female & $26 / 24$ & $25 / 21$ \\
Gestational age (weeks) & $39.1(1.6)$ & $39.2(1.5)$ \\
Birthweight (kg) & $3.3(0.6)$ & $3.4(0.6)$ \\
\hline
\end{tabular}

Table 3 Responders' antibody titres after third vaccine dose

\begin{tabular}{lllll}
\hline & $\begin{array}{l}10-80 \\
m U / m l\end{array}$ & $\begin{array}{l}\geqslant 80 \\
m U / m l\end{array}$ & $\begin{array}{l}\text { Total } \\
\text { Responders }\end{array}$ & $\%$ \\
\hline Preterm infants: & & & & \\
$\quad$ Stage 1 & 23 & 70 & & \\
$\quad$ Stage 2 & 11 & 52 & $156 / 176$ & 88.6 \\
Total & 34 & 122 & $43 / 46$ & 93.4 \\
Term infants & & 43 & \\
\hline
\end{tabular}

Data were analysed only for those who completed the study. All infants participating in the study were Caucasian, Jewish, or Arab.

The first vaccine was given to the preterm infants at a mean postnatal age of 1.9 (2.2) days and the term infants were all vaccinated immediately after birth. No clinically significant side effects attributable to the vaccine were noted in any of the infants. Side effects were monitored in the neonatal intensive care unit after the first and second vaccines. Mothers were questioned about side effects after the third vaccine dose a month later, when blood samples were drawn.

One hundred and fifty six preterm infants $(88.6 \%)$ responded to the vaccine (responders) and had protective antibodies against hepatitis B compared with 43 responders among the term infants $(93.4 \%)$. The preterm infants had a mean birthweight of $1.56(0.4) \mathrm{kg}$ and a mean gestational age of 31.9 (2.1) weeks. The term infants had a mean birthweight of $3.4(0.6) \mathrm{kg}$ and a mean gestational age of 39 (1.5) weeks.

One hundred and twenty two of the responders (out of 156), 70 in stage 1 and 52 in stage 2 , had a high titre of hepatitis B antibodies of more than $80 \mathrm{mU} / \mathrm{ml}$ (table 3) compared to all the term infants $(\mathrm{p}<0.05)$. In stage 2 the geometric mean antibody titre (GMT) was 469.1(486.2) $\mathrm{mU} / \mathrm{ml}$ whereas the term infants had a higher GMT of hepatitis B antibodies of 701.3 (450.6) $\mathrm{mU} / \mathrm{ml}(\mathrm{p}<0.03)$.

There were no differences in birthweight or gestational age between preterm infants enrolled in either stage or between responders and non-responders, or responders with a higher or lower titre of hepatitis B antibodies. No relation was found between the incidence of respiratory distress syndrome, sepsis, and jaundice and the immune response.

\section{Discussion}

The Committee on Infectious Diseases of the American Academy of Pediatrics stated in 1991 that "data on the effectiveness of hepatitis $B$ vaccine are not available for infants with birth weights less than 2000 grams." ${ }^{\text {7 }}$ At the initiation of the present study, the recommendation was to give the first hepatitis $B$ vaccine to all neonates soon after birth. ${ }^{7}$ An update in 1994 gave the following interim recommendations for hepatitis $\mathrm{B}$ vaccination of premature infants: "For premature infants with birth- weights of less than $2000 \mathrm{~g}$ born to HBsAgnegative women it may be advisable to delay initiation of hepatitis B vaccine until just before hospital discharge, provided the infant weighs $2000 \mathrm{~g}$ or more or until 2 months of age when other immunizations are given."

Our data suggest that preterm infants are capable of developing an adequate immune response to hepatitis B vaccine when immunised soon after birth. Similar to full term infants, close to $89 \%$ of our preterm infants had a protective level of hepatitis B antibodies 1-2 months after the third vaccine. Yet the level of antibodies was lower in the preterm infants than in the term infants. This could affect long term immunity and preterm infants may require a booster vaccination at an earlier age. The immune response of our preterm infants within each group was not related to birthweight, gestational age, or concurrent diseases.

Chawareewong's study ${ }^{1}$ was the first to report the immune response to hepatitis B vaccine in a small group of 14 preterm infants: $78.6 \%$ of the infants seroconverted when vaccinated at birth. Lau, in a study from Hong Kong, ${ }^{2}$ studied weight and age related effects on the response of preterm infants to hepatitis $B$ vaccine. Fifty seven preterm infants were vaccinated at birth or when reaching a weight of $1000 \mathrm{~g}$ (group 1), and 42 infants when reaching a weight of 2000 g. (group 2). After the third vaccine dose $79 \%$ and $91 \%$ seroconverted in groups 1 and 2, respectively. However, it is important to note that in Thailand and Hong Kong hepatitis $\mathrm{B}$ is endemic and many mothers are carriers of HBsAg, and, therefore, that these study population differed from ours.

Del Canho et al from Holland ${ }^{3}$ found that more than $95 \%$ of premature infants immunised soon after birth had hepatitis B antibody titres of $>10 \mathrm{mU} / \mathrm{ml}$ after the third vaccine dose. The better immune response rate obtained in their study may be attributable to the relatively big premature infants in their study. Another study from Thailand ${ }^{4}$ reported the immunogenicity of plasma derived hepatitis B vaccine in preterm infants and showed a $70 \%$ seroconversion rate after three vaccinations (at 0,1 , and 2 months after birth). A recent study from Philadelphia showed a similar seroconversion rate of $90 \%$ to hepatitis $\mathrm{B}$ vaccine even though in their study the first dose was given only at discharge. ${ }^{5}$ These various response rates to hepatitis $B$ vaccine assessed in different parts of the world may be due to differences in the genetically determined potential to develop an immune response in different populations of preterm infants. ${ }^{9}$

Interestingly, the immunogenicity of some other vaccines given to preterm infants soon after birth showed a lower response rate. One study determined the immune response to BCG vaccine given to premature infants at birth. ${ }^{10}$ A scar formation developed in only $68 \%$ of the preterm infants compared with $92 \%$ of the term infants. The difference in these results compared with those we found for the hepatitis B vaccine may reflect the varied 
maturity of different functions in the immune system. The immunogenicity and safety of inactivated polio vaccine in premature infants was determined during a small polio outbreak in Israel. ${ }^{11}$ At 1 month of age about $98 \%$ of the study infants had developed a protective titre of antibodies, showing that preterm infants can develop an immune response to polio vaccine even when vaccinated soon after birth.

Our data imply that hepatitis B vaccine is effective in most preterm infants when given soon after birth. In areas where screening mothers for HBsAg cannot routinely be done due to financial constraints, there is no reason to delay hepatitis $\mathrm{B}$ immunisation in preterm infants, thereby increasing their risk of acquiring hepatitis B from their unscreened mothers. Based on our findings it seems advisable to study the immune response of our preterm infants at 12 to 24 months of age. The incidence of infants with low hepatitis B antibody titres at a later age needs to be known to decide when a booster vaccine is required. The cost effectiveness of this approach requires verification. The new generation of hepatitis $B$ vaccines containing additional hepatitis $\mathrm{B}$ antigens, such as pre S1 and pre S2, may further improve their immunogenicity, perhaps eliminating the need for a fourth booster dose.

1 Chawareewong S, Jirapongsa A, Lokaphadana K. Immune response to hepatitis B vaccine in premature infants. Southeast Asian f Trop Med Public Health 1991;22:39-40.

2 Lau YL, Tam AYC, Ng KW, et al. Response of preterm infants to hepatitis B vaccine. $\mathcal{F}$ Pediatr 1992;121:962-5.

3 Del Canho R, Grosheide PM, Gerards LJ, Heijtink RA, Schalm SW. Hepatitis B vaccination and preterm infants. $\mathcal{f}$ Pediatr Infect Dis 1993;12:407-8.

4 Sanpavat S, Poovorawan Y, Chittinanad S, Bhongsvej S, Thaithumyanon F. Immunogenicity of Hepatitis B vaccine in premature infants. Southeast Asian $\mathcal{F}$ Trop Med Public Health 1994;25:93-5.

5 Kim SC, Chung EK, Hodinka RL, et al. Immunogenicity of hepatitis $B$ vaccine in preterm infants. Pediatrics 1997;99:534-6.

6 American Academy of Pediatrics. Report of The Committee on Infectious Diseases. Red Book. : Elk Groove Village, IL: American Academy of Pediatrics, 1991: 46.

7 Committee on Infectious Diseases, American Academy of Pediatrics. The report of the Committee on Infectious Diseases. 22 edn. American Academy of Pediatrics, Elk Groove Village, IL: 1991:238-55.

8 Committee on Infectious Diseases. Update on timing of hepatitis B vaccination for premature infants. Pediatrics 1994;93:403-4.

9 Alper CA, Kruskall MS, Marcus-Bagley D, et al. Genetic prediction of nonresponders to hepatitis B vaccine. $N$ Engl f Med 1989;321:708-12.

10 Sedaghatian MR, Kardouni K. Tuberculin response in preterm infants after BCG vaccination at birth. Arch Dis Child term infants after

11 Linder N, Yaron M, Hendsher R, et al. Early inactivated polio virus immunization in premature infants. $\mathcal{F}$ Pediatr 